EESTI NSV TEADUSTE AKADEEMIA TOIMETISED. XIII KÖIDE FCUSIKA-MATEMAATIKA- JA TEHNIKATEADUSTE SEERIA. 1964, NR. 4

ИЗВЕСТИЯ АҚАДЕМИИ НАУК ЭСТОНСКОН ССР. ТОМ ХІІІ СЕРИЯ ФИЗИКО-МАТЕМАТИЧЕСКИХ И ТЕХНИЧЕСКИХ НАУК. 1964, № 4

\title{
ОБ АЛГОРИТМАХ ПОСТРОЕНИЯ ЛОГИЧЕСКИХ СХЕМ
}

\author{
II. ХАНКО
}

\section{§1. Алгоритм для познания квазибесповторной реализуемости функции алгебры логики}

В статье $\left[{ }^{3}\right]$ показано, что для установления квазибесповторной реализуемости функции алгебры логнки $\left[{ }^{4}\right]$, заданной своей дизъюнктивной нормальной формой (д. н. ф.)

$$
\Re=\mathfrak{N}_{1} \vee \mathfrak{N}_{2} \vee \ldots \vee \mathfrak{N}_{t}
$$

приходится комбинировать члены этой д.н. ф. по три и исследовать квазибесповторную реализуемость д.н.ф., полученных таким образом. Если каждая трехчленная д.н. ф.

$$
\mathfrak{N}_{p} \vee \mathfrak{N}_{q} \bigvee \mathfrak{N}_{r} \quad(p=1,2, \ldots, t-2 ; q=2,3, \ldots, t-1 ; r=3,4, \ldots, t)
$$

имеет свойство $A$ абсолютно, то и д.н.ф. (1.1) имеет свойство $A$ абсолютно * и является формулой проводимости некоторой квазибесповторной схемы.

Трехчленная д.н.ф. (1.2), не имеющая свойства $A$ абсолютно, примет после выделения общих частей $\mathfrak{D}_{p q}, \mathfrak{D}_{p r}, \mathfrak{D}_{q r}$ ее слагаемых вид

$$
\mathfrak{A}_{p} \mathfrak{D}_{p q} \mathfrak{D}_{p r} \vee \mathfrak{A}_{q} \mathfrak{D}_{p q} \mathfrak{D}_{q r} \vee \mathfrak{H}_{r} \mathfrak{D}_{p r} \mathfrak{D}_{q r}
$$

в котором ни одна пара конъюнкций $\mathfrak{A}_{p}, \mathfrak{A}_{q}, \mathfrak{A}_{r}, \mathfrak{D}_{p q}, \mathfrak{D}_{p r}, \mathfrak{D}_{q r}$ не пересекается. В статье $\left.{ }^{3}\right]$ доказана следующая теорема:

Т ео рем а 1. Д.н.ф. (1.3) является формулой проводимости такой квазибесповторной схемы, в которий $\mathfrak{H}_{p}, \mathfrak{A}_{4}, \mathfrak{A}_{r}, \mathfrak{D}_{p q}, \mathfrak{D}_{p r}, \mathfrak{D}_{q r}$ означают последовательно соединенные ребра, тогда и только тогда, когда выполняется одно из условий

$$
\begin{array}{llll}
1^{\circ} \mathfrak{I}_{i j}=1 & \text { и } & \mathfrak{A}_{i} \mathfrak{I}_{i} \mathfrak{A}_{k}=0 \\
2^{\circ} \mathfrak{I}_{i}=1 & \text { и } & \mathfrak{A}_{i} \mathfrak{A}_{k}=0
\end{array}
$$

(индексы $i, j, k$ во всей статье не равны между собою и имеют значение или $p$, или $q$, или $r$ ).

* Свойство $A(a, b)$ д. н. ф. (1.1) состоит в следующем если хотя бы одна конъюнк. ция $\Re_{i}$ содержит булеву переменную $а$ и хотя бы одна конъюнкция $\Re_{i}(i \neq j)$ содержит булеву переменную $b(b \neq a)$, то ни одна конъюнкция не содержит $a b$. Д.н. ф. (1.1) нмеет свойство $A$ абсолютно, если ни одна пара булевых переменных в д. н. ф. (1.1) не нарушает свойства $A$. 
В настоящем параграфе исследуем вопрос о квазибесповторной реализуемости д.н.ф. (1.3) в более обшем случае, когда одна или две конъюнкции из $\mathfrak{A}_{p}, \mathfrak{V}_{q}, \mathfrak{N}_{r}, \mathfrak{D}_{p q}, \mathfrak{D}_{p r}, \mathfrak{D}_{q r}$ разделяются на две части, причем обе части означают в схеме последовательно соединенные ребра.

1. Разделим в д.н. ф. (1.3) одну из конъюнкций $\mathfrak{P}_{i}$ на две части, которые не имеют пересечений

$$
\mathfrak{A}_{i}=\mathfrak{U}_{i}^{1} \mathfrak{A}_{i}^{2} .
$$

Д.н. ф. (1.3) примет вид

$$
\mathfrak{U}_{i}{ }^{1} \mathfrak{U}_{i}{ }^{2}, \mathfrak{D}_{i j} \mathfrak{D}_{i k} \vee \mathfrak{U}_{j} \mathfrak{D}_{i j} \mathfrak{D}_{j k} \vee \mathfrak{U}_{k} \mathfrak{D}_{i k} \mathfrak{D}_{j k}
$$

т еорем а 2. Если одно из условий

$$
\begin{array}{ll}
1^{\circ} & \mathfrak{U}_{i}{ }^{2} \mathfrak{D}_{j k} \vee \mathfrak{U}_{j} \mathfrak{D}_{i k} \vee \mathfrak{A}_{i}{ }^{1} \mathfrak{U}_{k}=0 \\
2^{\circ} & \mathfrak{U}_{i}{ }^{2} \mathfrak{D}_{j k} \vee \mathfrak{U}_{i}{ }^{1} \mathfrak{U}_{j} \vee \mathfrak{U}_{i}{ }^{1} \mathfrak{U}_{k}=0
\end{array}
$$

вюпполнео, то д.н.ф. (1.4) является формулой проводимости такой квазибесповторной схемьи, в которой $\mathfrak{U}_{i}{ }^{1}, \mathfrak{U}_{i}{ }^{2}, \mathfrak{U}_{j}, \mathfrak{U}_{k}, \mathfrak{D}_{i j}, \mathfrak{D}_{i k}, \mathfrak{D}_{i k}$ означают последовательно соединенные ребра.

2. Пусть $\mathfrak{N}_{i}=\mathfrak{A}_{i}{ }^{1} \mathfrak{U}_{i}{ }^{2}$ и $\mathfrak{U}_{j}=\mathfrak{U}_{j}{ }^{1} \mathfrak{U}_{j}{ }^{2}$. Д.н.ф. (1.3) примет вид

$$
\mathfrak{U}_{i}{ }^{1} \mathfrak{U}_{i}{ }^{2} \mathfrak{D}_{i j} \mathfrak{D}_{i k} \vee \mathfrak{U}_{j}{ }^{1} \mathfrak{U}_{i}{ }^{2} \mathfrak{D}_{i j} \mathfrak{D}_{j k} \vee \mathfrak{A}_{k} \mathfrak{D}_{i k} \mathfrak{D}_{i k}
$$

Те о рем а 3. Если выполнено одно из условий

$$
\begin{aligned}
& 1^{\circ} \mathfrak{U}_{i}{ }^{2} \dot{D}_{j k} \vee \mathfrak{U}_{i}{ }^{2} \mathfrak{D}_{i k} \vee \mathfrak{U}_{i}{ }^{1} \mathfrak{U}_{i}{ }^{1} \mathfrak{U}_{k}=0 \\
& 2^{\circ} \quad \mathfrak{U}_{i}{ }^{2} \mathfrak{D}_{j k} \vee \mathfrak{U}_{i}{ }^{1} \mathfrak{A}_{i}{ }^{1} \vee \mathfrak{U}_{i}{ }^{1} \mathfrak{U}_{j}{ }^{2} \mathfrak{U}_{k} \mathfrak{D}_{i j}=0 \text {, } \\
& 3^{\circ} \quad \mathfrak{U}_{i}{ }^{1} \mathfrak{U}_{j}{ }^{1} \vee \mathfrak{U}_{i}{ }^{2} \mathfrak{U}_{i}{ }^{2} \vee \mathfrak{U}_{i}{ }^{1} \mathfrak{A}_{i}{ }^{2} \mathfrak{U}_{k} \mathfrak{D}_{i j} \vee \mathfrak{U}_{i}{ }^{2} \mathfrak{A}_{i}{ }^{1} \mathfrak{U}_{k} \mathfrak{D}_{i j}=0 \text {, }
\end{aligned}
$$

то д.н.ф. (1.5) является формулой проводимости такой квазибесповторной схемьи, в которой $\mathfrak{U}_{i}{ }^{1}, \mathfrak{U}_{i}{ }^{2}, \mathfrak{P}_{i}{ }^{1}, \mathfrak{U}_{j}{ }^{2}, \mathfrak{U}_{k}, \mathfrak{D}_{i j}, \mathfrak{D}_{i k}, \mathfrak{D}_{j k}$ означают последовательно соединенные ребра.

3. Пусть $\mathfrak{U}_{i}=\mathfrak{U}_{i}^{1} \mathfrak{U}_{i}{ }^{2}$ и $\mathfrak{D}_{j k}=\mathfrak{D}_{j k}{ }^{1} \mathfrak{D}_{i k}{ }^{2}$. Д.н.ф. (1.3) примет вид

$$
\mathfrak{U}_{i}{ }^{1} \mathfrak{U}_{i}{ }^{2} \mathfrak{D}_{i j} \mathfrak{D}_{i k} \vee \mathfrak{U} \mathfrak{D}_{i j} \mathfrak{D}_{i k}{ }^{1} \mathfrak{D}_{j k}{ }^{2} \vee \mathfrak{A}_{k} \mathfrak{D}_{i k} \mathfrak{D}_{j k}{ }^{1} \mathfrak{D}_{i k}{ }^{2}
$$

Т е орем а 4. Если выполнено одно из условий

$$
\begin{array}{ll}
1^{\circ} & \mathfrak{U}_{i}{ }^{2} \mathfrak{D}_{i k}{ }^{2} \vee \mathfrak{U}_{i}{ }^{1} \mathfrak{U}_{i} \vee \mathfrak{U}_{i}{ }^{1} \mathfrak{A}_{k} \mathfrak{D}_{i j}=0, \\
2^{\circ} & \mathfrak{U}_{i}{ }^{1} \mathfrak{D}_{i k} \vee \mathrm{V} \mathfrak{U}_{i}{ }^{2} \mathfrak{D}_{j k}{ }^{2} \vee \mathfrak{U}_{i}{ }^{1} \mathfrak{U}_{j} \mathfrak{H}_{k}=0,
\end{array}
$$

то д.н.ф. (1.6) является формулой проводимости такой квазибесповторной схемь, в которой $\mathfrak{A}_{i}{ }^{1}, \mathfrak{U}_{i}{ }^{2}, \mathfrak{A}_{i}, \mathfrak{U}_{k}, \mathfrak{D}_{i j}, \mathfrak{D}_{i k}, \mathfrak{D}_{i k}{ }^{1}, \mathfrak{D}_{i k}{ }^{2}$ означают последовательно соединенные ребра.

Д оказ ательство те орем 2, 3, 4. Легко видеть, что д.н.ф. (1.4) является формулой проводимости схемы 1, если выполнены условия $1^{\circ}$ или $2^{\circ}$ теоремы 2 ; д.н. ф. (1.5) является формулой проводимости схемы 2 , если одно из условий $1^{\circ}, 2^{\circ}, 3^{\circ}$ теоремы 3 выполнено; д.н. ф. (1.6) является формулой проводимости схемы 3, если выполнено одно из условий $1^{\circ}, 2^{\circ}$ теоремы 4. Схемы $1,2,3$ квазибесповторны, так как ни од- 
на пара из конъюнкций $\mathfrak{U}_{i}{ }^{1}, \mathfrak{U}_{i}{ }^{2}, \mathfrak{U}_{j}{ }^{1}, \mathfrak{U}_{j}{ }^{2}, \mathfrak{U}_{k}, \mathfrak{D}_{i j}, \mathfrak{D}_{i k}, \mathfrak{D}_{i k}{ }^{1}, \mathfrak{D}_{j k}{ }^{2}$ не пересекается. Теоремы доказаны.

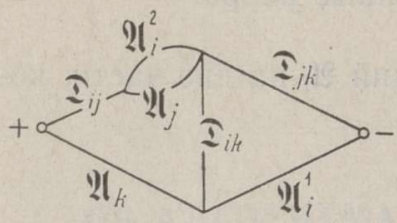

Схема 1.

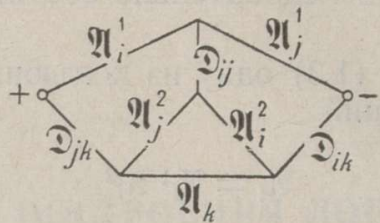

Схема 2.

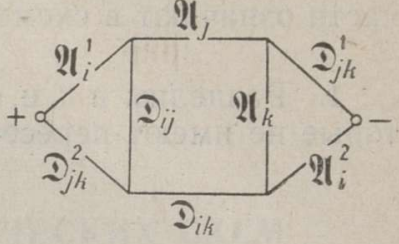

Схема 3.

Примеч ание 1. Когда выполнено. какое-либо из условий теорем $2,3,4$ или условие, составной частью которого является некоторое из условий теорем $2,3,4$, то д.н. ф. (1.4-6) могут являться формулами проводимости не только схем $1,2,3$, но и некоторых других схем. Применением алгоритма Трахтенброта-Пильчак $[1,2]$ к д.н.ф. (1.4-6) можно все эти схемы найти.

Итак, трехчленная д.н.ф. (1.3) квазибесповторно реализуема, если выполняется некоторое из условий теорем $1-4$. Теоремы $1-4$ допусқают объединение к следуюшей теореме:

Те орема 5. Если в д.н.ф. (1.3) выполняется одно из следующци услловий

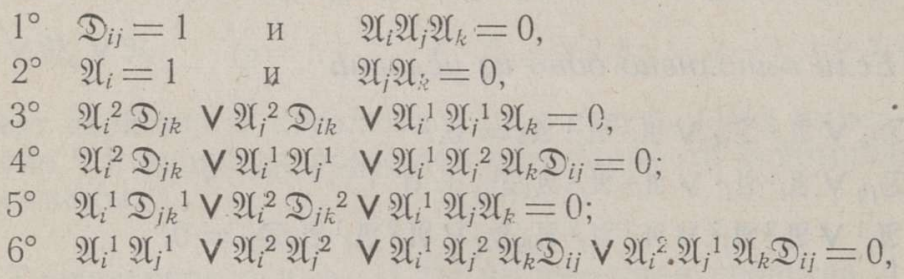

то д.н.ф. (1.3) является формулой проводимости такой квазибесповторной схемы, в которой $\mathfrak{U}_{i}{ }^{1}, \mathfrak{U}_{i}{ }^{2}, \mathfrak{U}_{i}{ }^{1}, \mathfrak{U}_{i}{ }^{2}, \mathfrak{U}_{k}, \mathfrak{D}_{i j}, \mathfrak{D}_{i k}, \mathfrak{D}_{i k}{ }^{1}, \mathfrak{D}_{i k}{ }^{2}$ означают последовательно соединенные ребра.

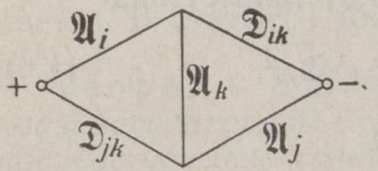

Схема 4.

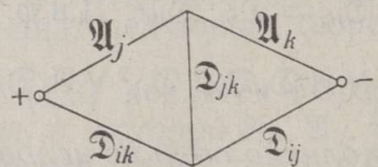

Схема 5.

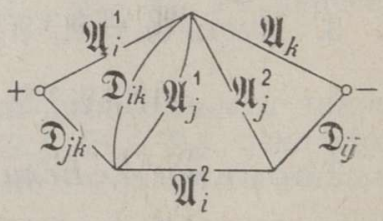

Схема 6.

При меч ан ие 2. Д.н.ф.

$$
\mathfrak{U}_{i} \mathfrak{D}_{i k} \vee \mathfrak{U}_{i} \mathfrak{D}_{j k} \vee \mathfrak{U}_{k} \mathfrak{I}_{i k} \mathfrak{D}_{i k} \vee \mathfrak{U}_{i} \mathfrak{A l}_{j} \mathfrak{U}_{k}
$$

является формулой проводимости для квазибесповторной схемы 4 и д.н.ф.

для схемы 5; если

$$
\mathfrak{D}_{i j} \mathfrak{D}_{i k} \vee \mathfrak{U}_{j} \mathfrak{D}_{i j} \mathfrak{D}_{j k} \vee \mathfrak{U}_{k} \mathfrak{D}_{i k} \mathfrak{D}_{j k} \vee \mathfrak{U}_{j} \mathfrak{U}_{k}
$$

$$
\mathfrak{U}_{i}{ }^{2} \mathfrak{D}_{j k} \vee \mathfrak{A}_{j}{ }^{2} \mathfrak{D}_{i k}=0,
$$


то д.н.ф.

$$
\mathfrak{U}_{i}{ }^{1} \mathfrak{U}_{i}{ }^{2} \mathfrak{D}_{i j} \mathfrak{D}_{i k} \vee \mathfrak{U}_{i}{ }^{1} \mathfrak{U}_{j}{ }^{2} \mathfrak{D}_{i j} \mathfrak{D}_{j k} \vee \mathfrak{U}_{k} \mathfrak{D}_{i k} \mathfrak{D}_{j k} \vee \mathfrak{U}_{i}{ }^{1} \mathfrak{U}_{j}^{1}
$$

является формулой проводимости для квазибесповторной схемы 2, д.н.ф.

$$
\mathfrak{U}_{i}^{1} \mathfrak{U}_{i}^{2} \mathfrak{D}_{i j} \mathfrak{D}_{i k} \vee \mathfrak{U}_{j}^{1} \mathfrak{U}_{j}{ }^{2} \mathfrak{D}_{i j} \mathfrak{D}_{j k} \vee \mathfrak{U}_{k} \mathfrak{D}_{i k} \mathfrak{I}_{j k} \vee \mathfrak{U}_{i}^{1} \mathfrak{U}_{k}
$$

- для схемы 6.

Сформулируем следующее прави,іо, нужное при нахождении тех трехчленов (1.2), которые не имеют свойства $A$ абсолютно:

П р а в и ло 1. Д.н.ф. (1.2) имеет свойство А абсолютно, если общие насти ее слагаемьх $\Re_{p}$ и $\Re_{q}$ удовлетворяют условию

$$
\mathfrak{D}_{p q}=\mathfrak{D}_{p r}
$$

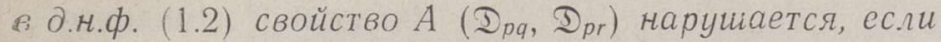

$$
\mathfrak{D}_{p q} \neq \mathfrak{D}_{p r}, \quad \mathfrak{D}_{p q} \neq 1, \quad \mathfrak{D}_{p r} \neq 1 .
$$

Действительно, после выделения общих частей слагаемых в д.н.ф.. (1.2) получим

$$
\mathfrak{R}_{p} \vee \mathfrak{N}_{q} \vee \mathfrak{N}_{r}=\mathfrak{U}_{0} \mathfrak{D}_{p q} \mathfrak{D}_{p r} \vee \mathfrak{U}_{q} \mathfrak{D}_{p q} \mathfrak{D}_{q r} \vee \mathfrak{U}_{r} \mathfrak{D}_{p r} \mathfrak{D}_{q r}
$$

(ни одна пара из конъюнкций $\mathfrak{A}_{p}, \mathfrak{A}_{q}, \mathfrak{U}_{r}, \mathfrak{D}_{p q}, \mathfrak{D}_{p r}, \mathfrak{D}_{q r}$ не пересекается). Если в (1.7)

то В д.н.ф.

$$
\mathfrak{D}_{p q}=\mathfrak{D}_{p r}=\mathfrak{I},
$$

$$
\mathfrak{N}_{p} \vee \mathfrak{R}_{4} \vee \mathfrak{N}_{r}=\mathfrak{A}_{p} \mathfrak{D} \vee \mathfrak{U}_{q} \mathfrak{D D S}_{q r} \vee \mathfrak{U}_{r} \mathfrak{D} \mathfrak{D}_{q r}=\left(\mathfrak{A}_{p} \vee \mathfrak{U}_{q} \mathfrak{D}_{q r} \vee \mathfrak{U}_{r} \mathfrak{D}_{q r}\right) \mathfrak{D}
$$

ни одна пара булевых переменных не может нарушить свойства $A$. Если в (1.7)

$$
\mathfrak{D}_{p q} \neq \mathfrak{D}_{p r}, \quad \mathfrak{D}_{p q} \neq 1, \quad \mathfrak{D}_{p r} \neq 1,
$$

то легко видеть, что свойство $A\left(\mathfrak{D}_{p q}, \mathfrak{D}_{p r}\right)$ нарушено.

Алгоритм для познания квазибесповторной реализуемости функции алгебры логики, заданной своей д.н.ф. (1.1), состоит в следующем:

а) Составим пары $\left(\Re_{p}, \mathfrak{N}_{q}\right)$ из членов данной д.н.ф. (1.1), выделим пбщую часть D $\mathfrak{D}_{p q}$ для каждой пары и впишем их в табл. 1. Если $\mathfrak{N}_{p}$ не пересекается с $\mathfrak{N}_{q}$, то $\mathfrak{D}_{p q}=1$. После составления табл. 1 переходим к пункту б).

б) Пользуясь методом сравнения величин $\mathfrak{D}_{p q}$ между собою, найдем те комбинации членов д.н.ф. (1.1) по три, которые не имеют свойства. A абсолютно:

Сравним $\mathfrak{D}_{p q}$ с $\mathfrak{D}_{p r}$. Если $\mathfrak{D}_{p q}=\mathfrak{D}_{p r}$, то д.н.ф. (1.2) имеет свойство $A$ абсолютно (правило 1) и мы переходим к рассмотрению следующей комбинации членов д.н.ф. (1.1) по три.

Если $\mathfrak{D}_{p q} \neq \mathfrak{D}_{p r}, \mathfrak{D}_{p q} \neq 1$ и $\mathfrak{D}_{p r} \neq 1$, то в д.н.ф. (1.2) нарушается свойство $A\left(\mathfrak{D}_{p q}, \mathfrak{D}_{p r}\right)$ (правило 1). Для выяснения квазибесповторной реализуемости д.н.ф. (1.2) переходим к пункту в). 
Таблича 1

\begin{tabular}{|c|c|c|c|c|}
\hline & $\Re_{2}$ & $\mathfrak{N}_{3}$ & $\Re_{t-1}$ & $\mathfrak{R}_{t}$ \\
\hline$\Re_{1}$ & $\mathfrak{D}_{12}$ & $\mathfrak{D}_{13}$ & $\mathfrak{D}_{1, t-1}$ & $\mathfrak{D}_{1 t}$ \\
\hline $\mathfrak{R}_{2}$ & & $\mathscr{D}_{23}$ & $\mathfrak{D}_{2, t-1}$ & $\mathfrak{D}_{2 t}$ \\
\hline$\dot{.}$ & & & & \\
\hline$\Re_{t-2}$ & & & $D_{t-2, t-1}$ & $\mathfrak{D}_{t-2, t}$ \\
\hline $\mathfrak{N}_{t-1}$ & & & & $\mathfrak{D}_{t-1, t}$ \\
\hline
\end{tabular}

Если $\mathfrak{D}_{p q} \neq \mathfrak{D}_{p r}$ и одна из них равняется единице, то приходится иметь в виду значение $\mathfrak{D}_{q r}$ : если $\mathfrak{D}_{q r}$ равняется $\mathfrak{D}_{p q}$ или $\mathfrak{D}_{p r}$, то д.н.ф. (1.2) имеет свойство $A$ абсолютно и мы переходим к рассмотрению следующей комбинации членов д.н.ф. (1.1) по три; в остальных случаях нарушается свойство $A\left(\mathfrak{D}_{p q}\right.$, $\left.\mathfrak{S}_{q r}\right)$ или $A\left(\mathfrak{S}_{p r}, \mathfrak{D}_{q r}\right)$ в д.н.ф. (1.2) и мы переходим к пункту в).

в) Отметим в д.н.ф. (1.2), которая не имеет свойства $A$ абсолютно, взятием в скобки обшие части конъюнкций $\mathfrak{N}_{p}, \mathfrak{R}_{q}, \mathfrak{N}_{r}$, так что д.н.ф. (1.2) примет вид

$$
\mathfrak{U}_{p} \mathfrak{D}_{p q} \mathfrak{D}_{p r} \vee \mathfrak{U}_{q} \mathfrak{D}_{p q} \mathfrak{I}_{q r} \vee \mathfrak{U}_{r} \mathfrak{D}_{p r} \mathfrak{D}_{q r}
$$

(ни одна пара из конъюнкций $\mathfrak{U}_{p}, \mathfrak{U}_{q}, \mathfrak{U}_{r}, \mathfrak{D}_{p q}, \mathfrak{D}_{p r}, \mathfrak{D}_{q r}$ больше не пересекается). Для познания квазибесповторной реализуемости д.н.ф. (1.3) будем поочередно проверять, выполнено ли некоторое из условий тепремы 5. Для этого переходим к пункту г).

г) Если оказывается, что в д.н.ф̣. (1.3)

$$
\mathfrak{I}_{i j}=1 \quad \text { и } \quad \mathfrak{U}_{i} \mathfrak{U}_{j} \mathfrak{U}_{k}=0 \text {, }
$$

то д.н.ф. (1.3) квазибесповторно реализуема; если

$$
\mathfrak{D}_{i j}=1 \text { и } \mathfrak{U}_{i} \mathfrak{U}_{j} \mathfrak{U}_{k} \neq 0 \text {. }
$$

то проверим, является ли $\mathfrak{U}_{i} \mathfrak{A}_{i} \mathfrak{U}_{k}$ членом д.н.ф. (1.1), так как д.н.ф. (1.7) квазибесповторно реализуема. В обоих случаях переходим к рассмотрению следующей комбинации членов д.н.ф. (1.1) по три. Если эти условия не выполнены, то переходим к пункту д).

д) Если оказывается, что в д.н.ф. (1.3)

$$
\mathfrak{A}_{i}=1 \text { и } \quad \mathfrak{P}_{j} \mathfrak{P}_{k}=0,
$$

то д.н.ф (1.3) квазибесповторно реализуема; если

$$
\mathfrak{A}_{i}=1 \text { и } \mathfrak{A}_{i} \mathfrak{A}_{k} \neq 0 \text {, }
$$


то проверим, является ли $\mathfrak{U}_{j} \mathfrak{Z}_{k}$ членом д.н.ф. (1.1), так как д.н. ф. (1.8) квазибесповторно реализуема. В обоих случаях переходим к рассмотрению следующей комбинации членов д. н. ф. (1.1) пю три. Если эти условия не выполнены, то переходим к пункту е).

е) Если оказывается, что в д.н.ф. (1.3)

$$
\mathfrak{U}_{i}^{2} \mathfrak{D}_{j k} \vee \mathfrak{U}_{j}^{2} \mathfrak{D}_{i k} \vee \mathfrak{U}_{i}^{1} \mathfrak{A}_{i}^{1} \mathfrak{U}_{k}=0
$$

то д. н. ф. (1.3) квазибесповторно реализуема; если

$$
\mathfrak{U}_{i}{ }^{2} \mathfrak{D}_{j k} \vee \mathfrak{U}_{i}{ }^{2} \mathfrak{D}_{i k}=0 \quad \text { и } \mathfrak{U}_{i}^{1} \mathfrak{U}_{i}^{1} \mathfrak{U}_{k}=0
$$

то проверим, является ли $\mathfrak{U}_{i}{ }^{1} \mathfrak{U}_{j}{ }^{1}$ или $\mathfrak{U}_{i}{ }^{1} \mathfrak{U}_{k}$ членом д. н. ф. (1.1), так как л.н.ф. (1.9) и (1.10) квазибесповторно реализуемы. При выполнении одного из этих условий переходим к рассмотрению следующей комбинации членов д.н.ф. (1.1) по три. Если эти условия не выполнены, то переходим к пункту ж).

ж) Если оказывается, что в д.н.ф. (1.3)

$$
\mathfrak{U}_{i}{ }^{2} \mathfrak{D}_{j k} \vee \mathfrak{U}_{i}{ }^{1} \mathfrak{U}_{j}{ }^{1} \vee \mathfrak{U}_{i}{ }^{1} \mathfrak{U}_{i}{ }^{2} \mathfrak{U}_{k} \mathfrak{D}_{i j}=0
$$

то д.н.ф. (1.3) квазибесповторно реализуема и мы переходим к рассмотрению следующей комбинации членов д. н. ф. (1.1) по три. Если это условие не выполнено, то переходим к пункту 3).

з) Если оказывается, что в д.н. ф. (1.3)

$$
\mathfrak{U}_{i}{ }^{1} \mathfrak{D}_{j k}{ }^{1} \vee \mathfrak{U}_{i}{ }^{2} \mathfrak{D}_{j k}{ }^{2} \vee \mathfrak{U}_{i}^{1} \mathfrak{U}_{j} \mathfrak{U}_{k}=0,
$$

то д.н.ф. (1.3) квазибесповторно реализуема и мы переходим к рассмотрению следующей комбинации членов д. н. ф. (1.1) по три. Если это условие не выполнено, то переходим к пункту и).

и) Если оказывается, что в д. н. ф. (1.3)

$$
\mathfrak{U}_{i}{ }^{1} \mathfrak{U}_{j}{ }^{1} \vee \mathfrak{U}_{i}{ }^{2} \mathfrak{U}_{j}{ }^{2} \vee \mathfrak{U}_{i}{ }^{1} \mathfrak{U}_{j}{ }^{2} \mathfrak{U}_{k} \mathfrak{D}_{i j} \vee \mathfrak{U}_{i}{ }^{2} \mathfrak{U}_{j}{ }^{1} \mathfrak{U}_{k} \mathfrak{D}_{i j}=0,
$$

то д.н.ф. (1.3) квазибесповторно реализуема и мы переходим к рассмотрению следующей комбинации членов д.н.ф. (1.1) по три. Если это условие не выполнено, то нам приходится повторить некоторые контакты в схеме, реализуюшей д.н.ф. (1.3) (см. в следующем параграфе алгоритм для определения повторений в сүеме).

к) Выполнение пунктов б) - и) чередуется до тех пор, пока рассмотрены все комбинации членов д.н.ф. (1.1) по три. Если все комбинации членов д.н.ф. (1.1) по три оказываются квазибесповторно реализуемыми, то и д.н. ф. (1.1) квазибесповторно реализуема.

Представленный нами алгоритм позволяет установить квазибесповторную реализуемость для широкого подкласса квазибесповторно реализуемых функций алгебры логики. Кроме того, он выделяет те комбинации членов д.н. ф. функции алгебры логики, которые «мешают» ее квазибесповторной реализуемости, и таким образом имеет значение для синтеза квазибесповторно нереализуемых функций. 


\section{$\S 2$. Алгоритм для определения повторений в схеме}

Для синтеза квазибесповторных схем разработан алгоритм Трахтенброта-Пильчак $\left[{ }^{1,2}\right]$. Этот алгоритм допускает обобщение на случай схем, содержащих повторения некоторых контактов. Распространение метода синтеза квазибесповторных схем на схемы, содержащие повторение, основано на том простом соображении, что если в некоторой схеме каждое явление некоторого контакта условно рассматривать как самостоятельный контакт, то она превратится в квазибесповторную. Выясним вышеуказанное при помощи следующего примера:

П р и м е р 1. Д.н. ф. функции алгебры логики

$$
f(x, y, z, w)=\bar{x} \bar{y} \bar{z} \vee x \bar{y} \bar{z} \bar{w} \vee \bar{x} \bar{y} \bar{w} \vee \bar{x} y \bar{z} \bar{w}
$$

является формулой проводимости схемы $7 a$.

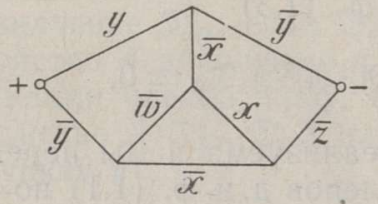

Cxema $7 a$.

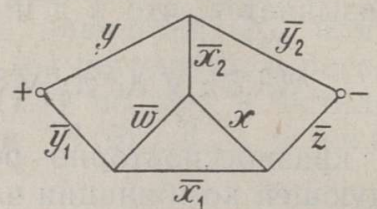

Схема 76.

Если в этой схеме повторные явления контактов $\bar{x}$ и $\bar{y}$ рассматривать как самостоятельные контакты и обозначать их разными символами $\bar{x}_{1}, \bar{x}_{2}$ и $\bar{y}_{1}, \bar{y}_{2}$, то она превратится в квазибесповторную схему 76 с формулой проводимости

$$
f_{1}=\bar{x}_{1} \bar{y}_{1} \bar{z} \vee x \bar{y}_{1} \bar{z} \bar{w} \vee \bar{x}_{2} \bar{y}_{1} \bar{y}_{2} \bar{w} \vee \bar{x}_{1} \bar{x}_{2} y \bar{z} \bar{w}
$$

В настоящем параграфе рассматривается случай, когда в д.н.ф. функции алгебры логики

$$
\mathfrak{N}=\mathfrak{N}_{1} \vee \Re_{2} \vee \ldots \vee \Re_{t}
$$

некая комбинация ее членов по три

$$
\mathfrak{A}_{p} \mathfrak{D}_{p q} \mathfrak{D}_{p r} \vee \mathfrak{U}_{q} \mathfrak{D}_{p q} \mathfrak{I}_{q r} \vee \mathfrak{U}_{r} \mathfrak{D}_{p r} \mathfrak{D}_{q r}
$$

не удовлетворяет условиям квазибесповторной реализуемости и таким образом «мешает» квазибесповторной реализации всей д. н. ф. (2.1). Для такого случая дается метод определения достаточных повторений в схеме и соответственного преобразования формулы проводимости схемы.

Сформулируем следующие два правила:

Пр а в ило 2. Д.н.ф. (2.2) можно всегда реализовать схемой, в которой повторяются дважды контакты $\mathfrak{D}_{i j} u \mathfrak{D}_{i k}$ u формулой проводимости которой является

$$
\mathfrak{U}_{i}\left(\mathfrak{D}_{i j}\right)_{1}\left(\mathfrak{D}_{i k}\right)_{1} \vee \mathfrak{A}_{j}\left(\mathfrak{D}_{i j}\right)_{2} \mathfrak{D}_{j k} \vee \mathfrak{A}_{k}\left(\mathfrak{D}_{i k}\right)_{2} \mathfrak{D}_{i k}
$$


ec. $2 u$

$$
\mathfrak{U}_{i} \mathfrak{U}_{j} \mathfrak{U}_{k} \mathfrak{D}_{i j}=0
$$

то д.н.ф. (2.2) реализуется схемой, в которой повторяется дванды контакт $\mathfrak{D}_{i j}$ и формулой проводимости которой является

$$
\mathfrak{U}_{i}\left(\mathfrak{D}_{i j}\right)_{1} \mathfrak{D}_{i k} \vee \mathfrak{U}_{j}\left(\mathfrak{D}_{i j}\right)_{2} \mathfrak{D}_{j k} \vee \mathfrak{U}_{k} \mathfrak{D}_{i k} \mathfrak{D}_{i k}
$$

Действительно, д. н. ф. (2.2) всегда реализуется схемой 8 с формулой проводимости (2.3); если

$$
\mathfrak{U}_{i} \mathfrak{U}_{j} \mathfrak{U}_{k} \mathfrak{D}_{i j}=0
$$

то она также реализуется схемой 9 с формулой проводимости (2.4).

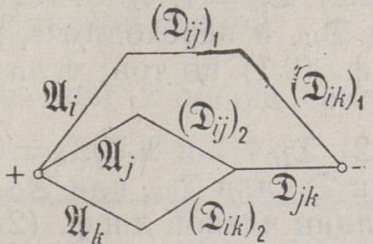

Схема 8.

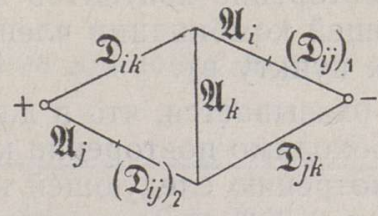

Схема 9.

Пусть в д. н.ф. (2.2)

$$
\mathfrak{D}_{i j}=1 \text {. }
$$

I. н. ф. (2.2) примет тогда вид

$$
\mathfrak{U}_{i} \mathfrak{D}_{i k} \vee \mathfrak{U}_{j} \mathfrak{D}_{j k} \vee \mathfrak{U}_{k} \mathfrak{D}_{i k} \mathfrak{D}_{j k}
$$

Пр а в и ло 3. Д.н.ф. (2.5) можно всегда реализовать схемой, в которой повторяется дваэды контакт $\mathfrak{D}_{i k}$ и формулой проводимости которой является

$$
\mathfrak{A}_{i}\left(\mathfrak{D}_{i k}\right)_{1} \vee \mathfrak{U}_{j} \mathfrak{D}_{i k} \vee \mathfrak{A}_{k}\left(\mathfrak{D}_{i k}\right)_{2} \mathfrak{D}_{i k}
$$

если в д.н.ф. (2.5)

$$
\mathfrak{U}_{j} \mathfrak{D}_{i b}=0
$$

то она реализуется также схемой, в которой повторяется дваюды контакт Dik $_{i k}$ формулой проводимости которой является

$$
\mathfrak{U}_{i}\left(\mathfrak{S}_{i k}\right)_{1} \vee \mathfrak{A}_{j} \mathfrak{D}_{j k} \vee \mathfrak{U}_{k}\left(\mathfrak{D}_{i k}\right)_{1}\left(\mathfrak{D}_{i k}\right)_{2} \mathfrak{D}_{j k}
$$

«น

$$
\mathfrak{U}_{i}\left(\mathfrak{D}_{i k}\right)_{1}\left(\mathfrak{D}_{i k}\right)_{2} \vee \mathfrak{U}_{j} \mathfrak{D}_{i k} \vee \mathfrak{U}_{k}\left(\mathfrak{D}_{i k}\right)_{1} \mathfrak{D}_{j k}
$$

Действительно, д.н. ф. (2.5) всегда можно реализовать схемой $10 a$ с формулой проводимости (2.6); если

$$
\mathfrak{U}_{i} \mathfrak{D}_{i k}=0
$$

то она реализуется еще и схемой 106 с формулой проводимости (2.7), или схемой 10 в с формулой проводимости (2.8).

8 ENSV TA Toimetised T-4 64. 


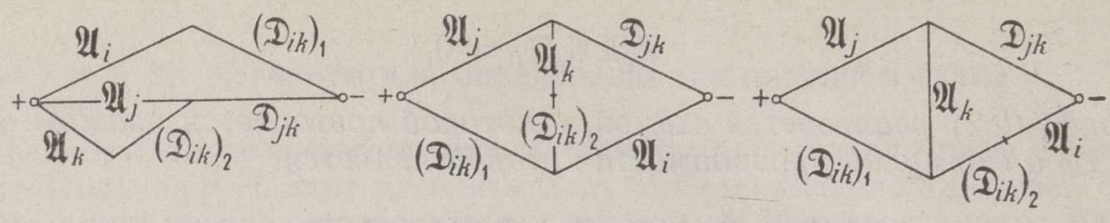

Схема $10 a$.

Схема 106 .

Схема 106 .

Алгоритм для определения достаточного числа повторений в схеме, реализующей д.н.ф. (2.1), состоит в следующем:

а) В ходе применения алгоритма установления квазибесповторной реализуемости выделяется комбинация (2.2) членов д.н.ф. (2.1) по три, которая не удовлетворяет условиям квазибесповторной реализуемости.

б) Если оказывается, что в д.н.ф. (2.2) $\mathfrak{D}_{i j}=1$, то отметим, что необходимо повторение контактов $\mathfrak{D}_{i k}$ или $\mathfrak{D}_{j k}$, и переходим к рассмотрению следующей комбинации членов д.н.ф. (2.1) по три; если $\mathfrak{D}_{i j} \neq 1$, то переходим к пункту в).

в) Если оказывается, что в д.н.ф. (2.2) $\mathfrak{I}_{i j} \neq 1$ и $\mathfrak{A}_{i} \mathfrak{A}_{j} \mathfrak{A}_{k}=0$, то отметим, что необходимо повторение контакта $\mathfrak{D}_{i j}$ или $\mathfrak{D}_{i k}$, или $\mathfrak{D}_{j k}$, и переходим к рассмотрению следующей комбинации членов д.н.ф. (2.1) по три; если $\mathfrak{D}_{i j} \neq 1$ и $\mathfrak{U}_{i} \mathfrak{U}_{j} \mathfrak{U}_{k} \neq 0$, то переходим к пункту г).

г) Если оказывается, что в д.н.ф. (2.2) $\mathfrak{D}_{i j} \neq 1$ и $\mathfrak{U}_{k} \mathfrak{D}_{i j}=0$, то отметим, что необходимо повторение контакта $\mathfrak{D}_{i j}$ или пары контактов $\left(\mathfrak{D}_{i k}, \mathfrak{D}_{i k}\right)$, и переходим к рассмотрению следующей комбинации членов д. н. ф. (2.1) по три; если $\mathfrak{D}_{i j} \neq 1$ и $\mathfrak{A}_{k} \mathfrak{D}_{i j} \neq 0$, то переходим к пункту д).

д) Отметим, что необходимо повторение пары $\left(\mathfrak{D}_{i j} \mathfrak{D}_{i k}\right)$ или $\left(\mathfrak{D}_{i j} \mathfrak{D}_{i k}\right)$, или $\left(\mathfrak{D}_{i k} \mathfrak{D}_{i k}\right)$, и переходим к рассмотрению следующей комбинации членов д.н.ф. (2.1) по три.

е) Выполнение пунктов а) - д) чередуется до тех пор, пока определены все те наборы контактов, повторение которых достаточно для реализации некоторой комбинации членов д. н. ф. (2.1) по три. Пусть этими наборами контактов будут

$$
\begin{aligned}
& \text { ( } \left.\mathfrak{F}_{11} \text { или } \mathfrak{F}_{12} \text { или } \mathfrak{E}_{13}\right) \\
& \left(\mathfrak{F}_{21} \text { или } \mathfrak{F}_{22} \text { или } \mathfrak{F}_{23}\right)
\end{aligned}
$$

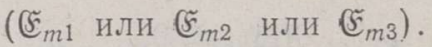

ж) Найдем минимальный набор контактов, повторение которых обеспечит реализуемость всей д.н.ф. (2.1): из наборов (2.9) образуем новые наборы контактов

$$
\begin{aligned}
& \left(\mathfrak{S}_{11}, \mathfrak{E}_{21}, \ldots, \mathfrak{S}_{m-1,1}, \mathfrak{E}_{m 1}\right) \\
& \left(\mathfrak{S}_{11}, \mathfrak{E}_{21}, \ldots, \mathfrak{E}_{m-1,1}, \mathfrak{E}_{m 2}\right)
\end{aligned}
$$

такие, что каждый из них содержит один элемент $\mathfrak{夭}_{\alpha \beta}$ из каждого набора (2.9). Повторение контактов в некотором наборе (2.10) обеспечит тогда реализуемость д. н. ф. (2.1). Так как некоторые из элементов (5аß) $1 \alpha=1,2, \ldots, m ; \beta=1,2,3)$ могут быть равными между собою, то наборы (2.10) будут содержать разные количества неравных элементов. Выбираем из наборов (2.10) те наборы, число неравных элементов которых буудет наименьшим. 
После выделения минимальных наборов из (2.10) переходим к пункту 3 ).

з) Преобразуем д.н. ф. (2.1), приписывая индексы к повторяющимся контактам.

Пр имер 2. Определить контакты, повторение которых достаточно для реализации д. н. ф. функции алгебры логики

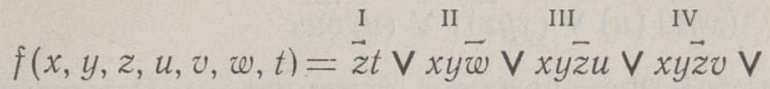

$$
\begin{aligned}
& \mathrm{v} \vec{x} u \vec{v} t \mathrm{v} \stackrel{\mathrm{vI}}{\mathrm{v}} \vec{\omega} t
\end{aligned}
$$

релейно-контактной схемой.

Составим табл. 2 общих частей членов данной д.н. ф.:

таблица 2

\begin{tabular}{|c|c|c|c|c|c|}
\hline & II & III & IV & V & VI \\
\hline I & 1 & $\bar{z}$ & $\bar{z}$ & $t$ & $t$ \\
\hline II & & $x y$ & $x y$ & 1 & $\bar{w}$ \\
\hline III & & & $x y \bar{z}$ & $u$ & $u$ \\
\hline IV & & & & 1 & 1 \\
\hline V & & & & & $u \bar{v} t$ \\
\hline
\end{tabular}

Следующие комбинации членов данной д. н. ф. по три не имеют свойства $A$ абсолютно, но удовлетворяют условиям квазибесповторной реализуемости (см. $\$ 1$ теорема 5):

$(I) \vee(I I I) \vee(V): \quad(\vec{z})(t) \vee x y(\vec{z})(u) \vee \bar{x}(u) \bar{v}(t)$

$$
\mathfrak{A}_{1}=1, \mathfrak{A}_{2}=x y, \mathfrak{U}_{3}=\overline{x v}
$$

(Удовл. усл. $2^{\circ}$ )

(I) $\vee(I V) \vee(V)$ :

$$
(\vec{z})(t) \vee x y(\vec{z}) \cup \vee \vec{x} u \vec{v}(t)
$$

$\mathfrak{D}_{23}=1, \mathfrak{A}_{1}=1, \mathfrak{A}_{2}=x y v, \mathfrak{A}_{3}=\bar{x} u \bar{v}$

(Удовл. усл. $1^{\circ}$ )

(I) $\vee(I V) \vee(V I)$ :

$$
(\vec{z})(t) \vee x y(\bar{z}) \cup \vee u \tilde{\tilde{w}}(t)
$$

$$
\mathfrak{D}_{23}=1, \mathfrak{P}_{1}=1, \mathfrak{P}_{2}=x y v, \mathfrak{P}_{3}=u \overrightarrow{\mathrm{v}}
$$

(II) $\vee(I V) \vee(V I): \quad(x y)(\vec{w}) \vee(x y) \bar{z} v \vee u \bar{v}(\bar{w}) t$

$$
\mathfrak{D}_{23}=1, \mathfrak{N}_{1}=1, \mathfrak{N}_{2}=\bar{z} \tau, \mathfrak{N}_{3}=u \bar{v} t
$$


(II) $\vee(\mathrm{V}) \vee(\mathrm{VI}): \quad x y(\bar{w}) \vee \bar{x}(u \bar{v} t) \vee(u \bar{v} t)(\bar{w})$

$$
\mathfrak{D}_{12}=1, \mathfrak{U}_{1}=x y, \mathfrak{U}_{2}=\bar{x}, \mathfrak{N}_{3}=1
$$

(Удовл. усл. $1^{\circ}$ )

(III) $\vee(I V) \vee(V): \quad(x y \bar{z})(u) \vee(x y \bar{z}) v \vee \bar{x}(u) \bar{v} t$

$$
\left.\mathfrak{D}_{23}=1, \mathfrak{U}_{1}=1, \mathfrak{N}_{2}=v, \mathfrak{U}_{3}=\overline{x v t} \quad \text { (Удовл. усл. } 1^{\circ}\right)
$$

(III) $\vee(I V) \vee(V I): \quad(x y \bar{z})(u) \vee(x y \vec{z}) v \vee(u) \bar{v} \bar{t} t$

$$
\mathfrak{D}_{23}=1, \mathfrak{N}_{1}=1, \mathfrak{A}_{2}=v, \mathfrak{A}_{3}=\vec{v} \overrightarrow{\omega t}
$$

(Удовл. усл. $\left.1^{\circ}\right)$

Следующие комбинации членов данной д.н.ф. по три не удовлетворяют условиям квазибесповторной реализуемости и требуют повторения некоторых контактов:

(I) $\vee($
(II) $\vee($ III) :

$(\bar{z}) t \vee(x y) \bar{w} \vee(x y)(\bar{z}) u$

$$
\mathfrak{D}_{12}=1, \mathfrak{P}_{1}=t, \mathfrak{P}_{2}=\bar{w}, \mathfrak{P}_{3}=u
$$

(Повт. $\bar{z}$ или $x y$ )

(I) $\vee$ (II) $\vee($ IV) :

$$
(\bar{z}) t \vee(x y) \bar{w} \vee(x y)(\bar{z}) v
$$

$$
\mathfrak{D}_{12}=1, \mathfrak{A}_{1}=t, \mathfrak{A}_{2}=\overline{\mathfrak{w}}, \mathfrak{A}_{3}=v
$$

(Повт. $\bar{z}$ или $x y$ )

(I) $\vee($ II) $\vee(V I)$ :

$$
\bar{z}(t) \vee x y(\bar{w}) \vee u \bar{v}(\bar{w})(t)
$$

$$
\mathfrak{D}_{12}=1, \mathfrak{N}_{1}=\bar{z}, \mathfrak{P}_{3}=x y, \mathfrak{P}_{3}=u \bar{v}
$$

(Повт. $\bar{\varpi}$ или $t$ )

(I) $\vee($ III) $\vee(V I)$ :

$$
(\bar{z})(t) \vee x y(\bar{z})(u) \vee(u) \bar{v} \bar{w}(t) \text {. }
$$

$$
\mathfrak{U}_{1}=1, \mathfrak{N}_{2}=x y, \mathfrak{N}_{3}=\overrightarrow{\text { चे }} \quad \text { (Повт. } \bar{z} u \text { или } \bar{z} t \text { или } u t \text { ) }
$$

(II) $\vee(I I I) \vee(V):$

$$
\begin{aligned}
& (x y) \bar{w} \vee(x y) \bar{z}(u) \vee \bar{x}(u) \bar{v} t \\
& \mathfrak{D}_{13}=1, \mathfrak{U}_{1}=\bar{w}, \mathfrak{U}_{2}=\bar{z}, \mathfrak{U}_{3}=\bar{x} \bar{t}
\end{aligned}
$$

(Повт. $и$ или ху)

(II) $\vee(I I I) \vee(V I):$

$$
(x y)(\bar{w}) \vee(x y) \bar{z}(u) \vee(u) \vec{v}(\bar{w}) t
$$

$$
\mathfrak{U}_{1}=1, \mathfrak{N}_{2}=\bar{z}, \mathfrak{U}_{3}=\overline{v t} \quad \text { (Повт. ит или хуи или хуш) }
$$

Остальные семь комбинаший членов д.н.ф. по три имеют свойство $A$ абсолютно (правило 1).

Образуем те наборы контактов, повторение которых обеспечит реализацию д.н.ф. (2.11):

$$
(\bar{z} u \bar{\varpi}), \quad(x y t u) \quad \text { и т. д. }
$$

Минимальным из этих наборов является (uㅜ). Преобразуем д.н.ф. (2.11), приписывая индексы к булевым переменным $\vec{z}, u, \vec{w}$ :

$$
f^{\prime}=\bar{z}_{1} t \vee x y \bar{w}_{1} \vee x y \bar{z}_{2} u_{1} \vee x y \bar{z}_{2} v \vee \bar{x} u_{2} \bar{v} t \vee u_{2} \bar{v} w_{2} t
$$

Д.н.ф. (2.11') функции алгебры логики $f^{\prime}$ квазибесповторно реализуема. Пегко увидеть, что она реализуется схемой 11 : 


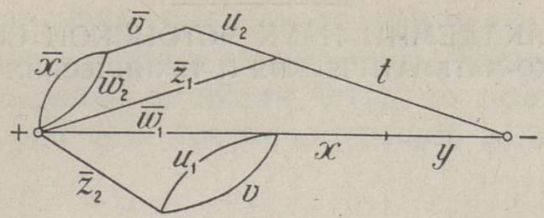

Схема 11.

Представленный алгоритм сопоставляет некоторой функции алгебры логики формулу проводимости некоторой квазибесповторной схемы, и таким образом распространяет метод синтеза квазибесповторных схем на схемы, содержащие повторения.

\section{ЛИТЕ РА Т У Р А}

1. П и льч а к Б. Ю., Пробл. кибернетикн, выг. 3. 95-122 (1960).

2. Тр ахтенброт Б. А., Тр. Матем. ин-та им. Стеклова АН СССР, 51, 226-269 (1958).

3. Х а н к о П., Изв. АН Эст. ССР. Сер. физ.-матем. и техн. наук, 12, № 3, $244-262$ (1963).

4. Я бл он ск и й С. В., Тр. Матем. ин-та им. Стеклова АН СССР, 51, 5-142 (1958).

Ннститут кибернетики

Академии наук Әстонской ССР
Поступила в редакцию

17. III 1964

\section{LOOGILISTE SKEEMIDE SÜNTEESIMISE ALGORITMIDEST}

\section{P. Hanko}

\section{Resümee}

Esitatakse kaks algoritmi, mis on vajalikud loogiliste skeemide sünteesimisel. Esimene algoritm eraldab antud loogilise funktsiooni disjunktiivses normaalkujus need liikmete kombinatsioonid, mis ei rahulda kvaasikordumisteta realiseeritavuse tingimusi. Juhul, kui selliseid kombinatsioone ei leidu, teeb algoritm kindlaks valemi realiseeritavuse kvaasikordumisteta skeemina. Teine algoritm määrab kindlaks vajalikud kordumised otsitavas skeenis.
Eesti NSV Teaduste Akadeemia
Küberneetika Instituut
Saabus toimetusse
17. III 1964

\section{ON SOME ALGORITHMS FOR A SYNTHESIS OF LOGICAL NETWORKS}

\section{P. Hanko}

\section{Summary}

Two algorithms which can be used for the synthesis of logical networks are presented. The first algorithm enables to determine those combinations of members of the disjunctive normal form of the logical function which do not satisfy the condition of quasinonrepetitionality. If aforementioned combinations do not exist, the algorithm allows to determine whether the logical function can be realized by a quasinonrepetitional network or not. The second algorithm determines the necessary repetitions in the network. 